\title{
REVISIONES
}

\section{El orden en la producción de conocimiento: Normatividades en la educación chilena en torno a diversidad*}

\author{
Order in the production of knowledge: Normativities in the Chilean education \\ around diversity
}

\author{
A ordem na produção de conhecimento: Normatividades na educação chilena \\ em torno da diversidade
}

\author{
Marcela Apablaza Santis
}

Universidad Austral de Chile, Valdivia, Chile.

Teléfono: (56) 63 2293510. Correo electrónico: marcela.apablaza@uach.cl

\begin{abstract}
RESUMEN
Trasladar la atención al lenguaje de diversidad y sus conceptos asociados, ha conducido a una forma de invisibilizar y neutralizar los cuestionamientos hacia regímenes de orden y normalización de los sujetos. En este artículo se analizan cuáles son los discursos presentes en la literatura científica chilena en torno a normalidad, diferencia y educación. Interesa exponer los discursos que circulan en el campo académico y analizarlos problemáticamente en relación a sus nociones, supuestos y perspectivas epistemológicas que subyacen a las ideas de diferencia, diversidad e inclusión. A partir de una mirada crítica sobre el actual escenario educativo político y tras el análisis de 45 artículos, el campo da cuenta de una producción de conocimiento dominada por los discursos de normalidad, lógicas de masculinización y concepciones naturalizadas respecto de las/los sujetos, cuyos efectos e implicancias se vinculan a la mantención de un proyecto neoliberal en el ámbito educativo.
\end{abstract}

Palabras clave: normalidad, diferencia, diversidad, educación, discurso.

\section{ABSTRACT}

Changing the focus to language of diversity and its associated concepts has led to an invisibilization and neutralization of any query towards the regimes of order and normalization of subjects. This article analyzes the discourse related to normality, diversity, difference and inclusion of scientific literature in the Chilean educational context. It is important to recall the academic discourse to analyze its notions, assumptions and the epistemological approaches which underlie the ideas of difference, diversity and inclusion. From a critical perspective toward the current political educational scenario and after the analysis of 45 articles, the field reveals a knowledge production dominated by discourses of normality, masculinization practices and naturalized conceptions of the subjects; the effects and implications are linked to the maintenance of a neoliberal project in education.

Key words: normality, difference, diversity, education, discourse.

\section{RESUMO}

Transladar a atenção para a linguagem da diversidade e seus conceitos associados, tem conduzido a uma forma de invisibilizar e neutralizar os questionamentos sobre regimes de ordem e de normalização dos sujeitos. Analisamse quais são os discursos presentes na literatura científica chilena sobre normalidade em torno dela mesma, da

Este artículo se enmarca dentro del Proyecto Anillos en Ciencias Sociales y Humanidades, "Normalidad, Diferencia y Educación" (SOC1103), el cual cuenta con el financiamiento del Programa de Investigación Asociativa-Comisión Nacional para la investigación Científica y Tecnológica (PIA-CONICYT). 
diferença e da educação. Interessa, aqui, a exposição dos discursos que circulam no campo acadêmico e a análise deles, problematicamente, no que diz respeito às suas noções, pressupostos e perspectivas epistemológicas subjacentes às ideias de diferença, diversidade e inclusão. A partir de um olhar crítico sobre o atual cenário político-educacional e análise de 45 artigos, o campo aponta para a produção de conhecimento dominado por discursos de normalidade, lógicas de masculinização e concepções naturalizadas sobre os sujeitos, cujos efeitos e implicações estão ligados à manutenção de um projeto neoliberal no âmbito da educação.

Palavras chave: normalidade, diferença, diversidade, educação, discurso.

\section{LO PROBLEMÁTICO: LA VIGENCIA NEGADA DE LA NORMALIDAD Y SU ARTICULACIÓN CON DIFERENCIA}

Amparados en términos como diversidad, diferencia e inclusión, tanto la producción de conocimiento como el campo práctico han adoptado dicho lenguaje bajo los principios de equidad y de justicia social como un modo de hacer frente a la normalidad (Ahmed, 2007; Baez, 2000). No obstante, diversos autores/autoras reclaman y advierten que trasladar la atención al lenguaje de diferencia y a sus conceptos asociados, nos ha conducido a invisibilizar y neutralizar los cuestionamientos hacia regímenes de orden y normalización de las/los sujetos (Baez, 2000; Davis, 1995; Kumashiro, 1999; Matus \& Infante, 2011), discursos que hasta ahora se mantienen en un estatus incuestionable.

En un escenario de globalización y neoliberalización, dichas temáticas han cobrado más relevancia, pues la educación ha sido valorada como uno de los componentes claves para el desarrollo y la producción económica, por lo que problemáticas de exclusión, desigualdad social y segregación son puestas en un nivel prioritario dentro de las agendas gubernamentales (Dussel, 2004), y así los países enmarcados en lineamientos propuestos por organismos internacionales, elaboran una serie de políticas sociales con el objeto de avanzar hacia dicho propósito y, de paso, acceder a ciertos beneficios (Matus e Infante, 2011; Popkewitz \& Lindblad, 2000).

La literatura internacional nos presenta un prolífero y vasto campo de investigación. Se hallan así diversas corrientes, tales como necesidades educativas especiales (NEE) (Slee, 2008, Ainscow \& Sandill, 2010), interculturalidad (Bryan, 2010; Duchatsky, 1996; Palailogou \& Faas, 2012), inclusión social (Popkewitz \& Lindblad, 2000), estudios de raza (Baez, 2003; Kamp \& Mansouri, 2010), género y sexualidad, por mencionar algunas.

Popkewitz y Lindblad (2000) analizan la producción de conocimiento desde dos perspectivas: la problemática de equidad y de conocimiento. La primera abarca aquellos estudios preocupados de constatar, más que de problematizar, la mantención de cierto régimen de orden. Desde un foco aparentemente neutral, comprenden la inclusión bajo una mirada binaria, contrapuesta a la exclusión (que hay que eliminar) y valorada como una meta a alcanzar. Aquí encontramos estudios que generan evidencias sobre lo que falta, en cuyo análisis se emplean discursos acríticos y aparentemente neutrales, respecto de dichos principios y nociones asociadas.

En cambio, la problemática de conocimiento cuestiona el conocimiento naturalizado y arraigado que regula cierto orden normativo en el campo educacional, ya sea en sus políticas, modos de administración y prácticas pedagógicas. Desde perspectivas problematizadoras, estos estudios cuestionan la producción de conocimiento tradicional visto como discursos/ conocimientos que reafirman y perpetúan un régimen de orden esencialista y normativo que configuran una idea de sujeto universal. 
Por su parte, Chile ha mantenido un ritmo de avance dinámico en sus políticas educacionales, especialmente en el ámbito de educación especial (Fundación Chile, 2013; López et al., 2014). No obstante, se desconocen los supuestos y perspectivas epistemológicas que sustentan la producción de conocimiento que nutren en alguna medida estas políticas, lo que nos permitiría comprender parcialmente las condiciones de producción de las diferencias a nivel educativo y la mantención privilegiada de lo normativo. A partir de esta problemática, me interesa indagar en la literatura científica vinculada a temáticas de normalidad, desde los conceptos de diversidad/diferencia/inclusión en el ámbito educativo chileno, con el propósito de exponer los discursos que circulan y analizarlos problemáticamente en relación a sus nociones, supuestos y perspectivas epistemológicas.

Organizo este artículo en tres movimientos: inicialmente, expongo los elementos procedimentales para delimitar el corpus; posteriormente presento las premisas teóricas que sustentan el modo de aproximación analítica; y, finalmente, analizo y discuto críticamente las distinciones discursivas halladas en el campo de conocimiento, con el objeto de problematizar las implicancias que traen consigo tales discursos.

\section{FRONTERAS PROCEDIMENTALES}

En base a los criterios de inclusión/exclusión que presento a continuación, la delimi-tación del corpus y su posterior análisis lo elaboré de acuerdo al siguiente procedimiento.

En un primer momento, efectuamos la examinación de bases de datos online (WoS, Scopus, Scielo, ERIC), examinación de revistas nacionales e internacionales y rastreo por autores. Para ello, se emplearon motores de búsqueda, focalizando sobre términos como: diversity, inclusion, difference, education, Chile, y sus términos relacionados: inclusive education, special educational needs, identity, normalcy, gender, etnicity. En esta etapa se obtuvieron 112 artículos. La revisión de artículos y abstracts permitió reducir el corpus a 88 artículos en un primer barrido, para finalmente seleccionar 45 artículos, los que conformaron el corpus de análisis central.

Puesto que mi interés trasciende a los resultados y metodologías reportadas, utilizo la perspectiva de análisis de discurso como herramienta de intelección para examinar los discursos que circulan en el campo, tanto de las/los autores, como de los hallazgos resultantes de sus investigaciones.

Desde una mirada foucaultiana, comprendo al discurso como práctica social, como unidad básica de la configuración social, donde este pasa a ocupar un lugar crucial para la comprensión de la vida social, para la producción de conocimiento, la constitución del sujeto y la formación de las instituciones sociales, más allá del sustrato material que lo soporta y donde se imbrican otros dos componentes centrales, las relaciones de poder y el saber-conocimiento. Complementariamente, desde la perspectiva del Análisis Político del Discurso (APD), asumo ciertas cualidades centrales de este, su pluralidad, apertura, movilidad, relacionalidad e inconmensurabilidad (Buenfil, 1994; Seidman, 2004), que en palabras de (Foucault, 2011) correspondería a "las prácticas que forman sistemáticamente los objetos de que hablan" (p. 68).

El corpus de análisis fue delimitado de acuerdo a los criterios de inclusión/exclusión previamente establecidos. De acuerdo a ellos seleccioné artículos de investigación, revisiones de literatura y artículos teóricos de origen chileno, en idioma inglés o español, 
publicados entre el año 2000-2013. Se incluyeron artículos referidos a temáticas de diferencia/diversidad/inclusión en el ámbito educacional en todos sus niveles (educación pre-escolar, escolar o primaria y secundaria y educación superior o terciaria).

\section{NORMALIDAD, DIFERENCIA, EDUCACIÓN: VISIBILIDAD Y SUBVERSIÓN}

La normalidad nos lleva a pensar en un orden, en un sistema de regularidades que de un modo entretejido conforman y a la vez se forman a través de un conjunto de reglas, leyes y determinaciones, aparentemente fijas y totalitarias, que dan paso a ciertos modos de organizar nuestra visión respecto del mundo y de su funcionamiento. Esto trae consigo ciertos supuestos acerca de cómo comprendemos el mundo, "La realidad", específicamente si se trata de una construcción social o algo externo capturable (Haraway, 1995; Scott, 1992), lo social y las/los sujetos.

Por su parte, el lenguaje de diversidad/diferencia/inclusión también trae consigo una serie de supuestos vinculados a conceptos de particularidad y universalidad. El desarrollo del conocimiento ha abordado tales principios con cierto énfasis ya sea en uno, en el otro o en ambos. Así, desde una mirada cartesiana vemos cómo lo universal prima sobre lo particular, donde el conocimiento persiste en la búsqueda de la totalización y objetivación de la realidad. En cambio, actualmente vemos la manera en que el pensamiento moderno ha estrechado la relación universalidad-particularidad en base a un planteamiento que si bien promueve la necesidad de universalización, este distingue a lo particular como un valor cuando se trata de las/los sujetos (Bordo, 1986; Haraway, 1995; Laclau, 1996). Diversidad, un término que ha sido ampliamente acogido por el campo político, académico y social, es uno de los conceptos que se ha atribuido al énfasis en lo particular como un principio que rescata los atributos individuales de cada sujeto.

Expuesto el marco que sustenta el lenguaje de diversidad, me propongo cuestionar dicho término por sus orígenes, usos y fines. En esta línea argumentativa, diferentes propuestas teóricas sitúan el término diversidad en el campo de la biología y, específicamente, en la teoría de la evolución (Baez, 2004), la cual sostiene la condición de un conjunto de atributos individuales que permiten distinguir las diferencias sociales, y por tanto las sitúan en un plano natural e invariable (Baez, 2000; Baez, 2003; Baez, 2004), por lo que su uso dentro de las políticas se orientaría más bien a buscar medidas compensatorias o asimilacionistas centradas en "aceptar", "respetar" y "tolerar" tales distinciones, más que en afianzar una transformación social.

Por otra parte, no podemos obviar la relación estrecha y articulada que se ha forjado entre términos como diversidad e inclusión y neoliberalismo, entendido este último como un proyecto transversal a lo económico y a lo político (Duggan, 2004; Fairclough, 2000; Fairclough, 2013; Rizvi \& Lingard, 2013), que define la vida cotidiana, lo social y por tanto se extiende al campo educativo como espacio de actuación y despliegue clave. Así vemos cómo las políticas afirmativas, enmarcadas en principios de equidad y justicia social, adoptan el lenguaje de diversidad como estandarte para mantener el status quo económico-social, bajo el reconocimiento de la aceptación de "lo diverso", sin posibilidad alguna de que dicha diversidad pueda cambiar su posición de ser "lo diferente". En este sentido, proponentes como Ahmed (2007), Infante et al. (2011) y Baez (2000) nos exponen los intereses en juego ante el uso de términos como diversidad e inclusión, donde la 
neutralidad del término y su vinculación a ciertos principios y valores toman distancia y apartan la discusión y reflexión desde uno de los principales ejes de origen, me refiero a las relaciones de poder en juego y a su implicancia en la organización de la estructura social. De este modo, la subalternización, la opresión y la otrarización de las diferencias quedan relegadas a un lenguaje de mayor confrontación y de menor aceptación social, por lo que el lenguaje de inclusión y de diversidad pasa a tomar un lugar de anhelo social.

Desde este punto, consideramos que hablar de diferencia nos refiere a un conjunto de supuestos sustentados en perspectivas distintas que hablar de diversidad. Referirse a diferencia es asumir que tales atributos son el resultado de una serie de textualidades, es decir, de construcciones discursivas, culturales acerca de las/los sujetos, que poseen un carácter contextual, parcial, no universal, no fijo y, por tanto, modificable (Baez, 2004). Baez (2000), en su Teoría de la diferencia, cuestiona el carácter natural que se le ha otorgado y señala la importancia de estudiar y problematizar sus orígenes, condiciones de mantención y fines para los cuales las diferencias pueden ser producidas y reforzadas.

Poner atención a los discursos de normalidad, supuesto que acompaña este trabajo, es trastocarlo y cuestionarlo para así devolverle su carácter parcial y no trascendental, por lo que interesarse en los discursos que circulan en torno a diferencia/diversidad/inclusión, nos permitiría distinguir los sistemas de razón que han modelado y fijado la aparente inmovilidad de los regímenes normativos. Es ante estos fines que me propongo elucidar los supuestos, perspectivas epistemológicas y nociones acerca de diferencia/diversidad/ inclusión, que circulan en el campo de la literatura científica.

Para ello, he organizado el corpus analizado bajo tres temas orientadores: Nociones y supuestos que circulan en el campo; objetos de estudio y perspectivas metodológicas presentes.

\subsection{LA NORMALIDAD NEGADA Y AFIRMADA: NOCIONES Y SUPUESTOS EN CIRCULACIÓN}

Parto de la premisa de que el conjunto de significados, términos y lenguajes empleados en la producción de conocimiento, constituyen formaciones discursivas que en su modo entretejido y puesto a circular en el campo, definen las relaciones que se establecen, sus regularidades y fijaciones temporales, las que conforman ciertos sistemas de organización que rigen en el mundo social en un momento determinado (Foucault, 2011).

Bajo este prisma, los artículos analizados conforman un marco discursivo compuesto por términos como diversidad, inclusión, diferencia, igualdad, justicia social, democracia, derechos, interculturalidad y multiculturalidad, entre los más recurrentes. No obstante, enunciarlos en un modo nominal no nos conduce a mayores aclaraciones. Lo que me gustaría distinguir es su modo de vinculación y articulación entre sí.

En su mayoría, estos estudios asumen como principios de base la igualdad, democracia, justicia social y perspectiva de derecho, alineados con discursos oficiales propuestos por organismos internacionales. Esta articulación discursiva, haciendo referencia a Baez (2004), obedece a las implicancias y supuestos que acompañan los estudios de diversidad, donde este lenguaje, en su apariencia, asume una condición de agencia social, no obstante en su carácter eclipsado, trae consigo un discurso determinista y naturalizado respecto de las/los sujetos, en el que las diferencias, más que cuestionarlas en su condición de producción, se centran en la "aceptación" o "tolerancia", para lo cual hace uso de principios como democracia, igualdad y justicia social. 
Asimismo, Ahmed (2007) nos permite elucidar la vinculación entre diversidad e igualdad, donde el uso de diversidad se debe al desgaste que han tenido términos como igualdad y justicia social, por su carácter confrontacional y rupturista. Por lo que hablar de diversidad tendría más posibilidades de éxito que las que no lograron sus términos antecesores. Esto nos conduce a pensar que el uso de diversidad acompaña un proyecto político, que más que ser neutral y emancipador, es mantenedor y reproductor del status quo de la estructura social.

En un modo más específico, diversidad a ojos de las/los autores analizados, es vista como una riqueza y un valor (Caijao, 2005; Elizalde, 2005; Fernández, 2001; Ibáñez, 2010; Julio, 2009; Magendzo, 2005; Tenorio, 2005), cuyo punto de convergencia es su crítica a lo homogéneo, donde se reconoce la uniformidad como característica constitutiva de nuestro sistema educativo, lo que para la mayoría de las/los proponentes constituiría una tecnología de control, cuya institución eje es la escuela. En esta línea discursiva, el lenguaje de diversidad adquiere un valor aparentemente liberador o emancipador, pues se asume que una visión pluralista del mundo conduciría a eliminar la exclusión/opresión de ciertos grupos que no cumplen con el régimen normativo, no obstante, tales discursos caen en serias contradicciones a ojos de otros autores, quienes cuestionan sus propósitos emancipadores y los sitúan como reproductores de una agenda neoliberal (Infante \& Matus, 2009; Matus \& Infante, 2011).

Asimismo, diversidad es descrita como algo complejo, posible de conocer, como un desafío (Elizalde, 2005), necesario de aceptar y legitimar (Ibáñez, 2010; Becerra, 2012; Díaz \& Druker, 2007), como un medio (Schmelkes, 2005), posible de abordar y manejar (Magendzo, 2000; Prieto, 2001). Para algunos esta dicotomía diversidad/homogeneidad es comprendida como un medio de categorización con el objeto de alcanzar cierta inteligibilidad, en este caso, de las/los sujetos. En este sentido, resulta valioso recordar lo planteado por Deleuze (2006), quien tensiona esta relación respecto del desarrollo del conocimiento bajo premisas modernas del orden taxonómico como un mecanismo para aprehender la realidad. Esto nos permite comprender la regularidad discursiva presente en los artículos, que en su mayoría desarrollan análisis y reflexiones en torno a lo que es $L A$ diversidad, LA diferencia, como Un singular/trascendental y totalitario objeto de estudio.

Pensadoras feministas como Susan Bordo (1986) y Elizabeth Grosz (1995) nos muestran cómo la producción de conocimiento, a partir del giro cartesiano, ha sido desarrollada en base a y dominada por los imperativos de la masculinización, cuyo elemento central es la disociación mente/cuerpo, razón/percepción, certeza/caos, y donde la completitud o mirada desde la complejidad (asociado a lo femenino), es vista como carente de significado, ausente de rigurosidad y validez, por tanto deslegitimada. Para estas autoras, dicha supremacía masculina no permite cuestionar y repensar los modos de comprender el mundo y cuyas implicancias, en esta racionalización del conocimiento y abandono de la mirada integrada, conducen a conocer el mundo bajo Una forma y acceder a La respuesta, a la verdad. Así, La diversidad pasa a ser considerada un objeto de estudio, las/los sujetos considerados desde un cierto orden como "Otros"/"Otras" se transforman en la otredad, en un asunto a ser medido, analizado e intervenido. Frecuentemente, en la literatura podemos hallar frases como 'la diversidad es un desafío complejo para las escuelas', lo que corrobora esta idea de una diversidad necesaria a ser abordada, manipulada e intervenida para ser incluida.

La mirada binaria normalidad/anormalidad conforma una regularidad presente en el campo, donde lo diferente o diverso permanece subsumido a la primacía de lo 
normativo. Aun cuando las/los autores proponen superar el paradigma normativo, los análisis expuestos contraponen tal propósito, y más bien terminan por reafirmarlo, lo que conduce a la mantención de una visión centro/margen que muchos de los estudios parten cuestionando (Caijao, 2005; Elizalde, 2005; Fernández, 2001; Schmelkes, 2005). Así podemos encontrar estudios que articulan íntimamente inclusión y NEE (Díaz et al., 2007; Sánchez et al., 2008; Tenorio, 2011). Esto da cuenta de la prevalencia de una mirada tradicional, acrítica respecto de los discursos de normalidad, donde se emplean lenguajes bajo una visión biologicista proveniente del modelo médico, centrada en el diagnóstico y por tanto configuradora de discursos más bien esencializadores de las/los sujetos.

Otras autoras, comprenden la diversidad como un discurso que va más allá de lo totalizante y unificador, como una postura epistémica, ética y política que rescata la totalidad y complejidad del sujeto (Matus, 2005) inmerso en un contexto determinado, y donde la alteridad es requerida como condición necesaria del sistema educativo, de lo contrario se llega a su folklorización (Magendzo, 2005). Desde esta perspectiva, diversidad es vista como una construcción discursiva de carácter contingente, donde convergen ciertas relaciones de poder que configuran ciertos procesos de subjetivación que, de acuerdo a los usos que algunos autores/autoras reclaman, configurarían ciertas subjetividades y su subsecuente subalternización, lo que nos lleva a pensar de qué manera los usos del lenguaje de diversidad contribuyen a la textualización de los sujetos, inscripción de los cuerpos y su configuración estigmatizada.

Los discursos sobre diferencia, en su mayoría, son subsumidos bajo el lenguaje de diversidad, lo que se refleja en la idea de diferencia articulada con ciertos marcadores de identidad (etnia, género, clase social, ritmos de aprendizaje), delimitándolos como un hecho existente, ligado al dato empírico. Ciertamente, las diferencias se asumen como un atributo/condición de ciertos sujetos, identificados bajo una posición de opresión o subyugación ante aquellos que ocupan un lugar privilegiado, por tanto configura una visión naturalizada, un rasgo constitutivo del ser, en referencia a una normalidad "existente" y plenamente vigente.

Finalmente, los estudios que hacen referencia a las temáticas de inclusión, mayoritariamente se centran en analizar aquello que falta para alcanzarla, sin cuestionar las bases del término, excepto Infante (2010) quien en referencia a la formación inicial, plantea la necesidad de cuestionar aquello que se define en torno a la normalidad, a la referencia de aquella centralidad que determina el ideal de estudiante, y en consecuencia se configura como un artefacto de poder que restringe cualquier práctica inclusiva posible. La tensión que aquí se produce es justamente lo que Popkewitz \& Lindbland (2000) argumentan, por un lado, a través de un enfoque que aborda la inclusión desde la problemática de equidad, situándola como una meta factible, en tanto su contraparte, la exclusión, sea erradicada; y por otra parte, encontramos perspectivas que elaboran la inclusión desde la problemática del conocimiento, posicionándola en un lugar de cuestionamiento en relación a sus bases y condiciones de producción y en una mirada continua, mas no contraria a la exclusión.

\subsection{OBJETO DE ESTUDIO: USOS Y FINES DEL CONOCIMIENTO PRODUCIDO}

Las/los autores sostienen que los discursos de diversidad circulan bajo intereses políticoseconómicos neoliberales y de globalización (Caijao, 2005; Infante, 2009; Matus, 2005; Matus \& Infante, 2011), cuya expresión se evidencia en su demanda por valorar la 
diversidad cultural como riqueza del patrimonio intangible de cada país (Caijao, 2005). Así, la diversidad se convierte en un desafío virtuoso y efectivo (Caijao, 2005; Fernández, 2001; Magendzo, 2000; Schmelkes, 2005), cuyo sujeto portador se convierte en un objeto posible de ser transformado desde su condición de desventaja anterior, a una ventaja para el progreso y el desarrollo del país. En contraposición, autoras como Matus e Infante (2011) cuestionan su abordaje fragmentario y naturalizado y sostienen que tales discursos neutralizan los asuntos de poder en las políticas educativas mercantilizantes, donde los valores de desempeño son celebrados y puestos como un desafío a cumplir.

Otra relación usualmente encontrada en el cuerpo de la literatura es la vinculación entre diversidad, inclusión y NEE. Si bien las/los autores no hacen referencia a ello, es necesario detenernos y analizar tal articulación, pues no podemos obviar el triunfo y consolidación que han tenido los discursos de diversidad en Chile de la mano de las políticas de educación especial. Desde una mirada del análisis político del discurso, esta relación entre significantes, podría ser vista como una operación de condensación, pues aun cuando cada concepto tiene un significado por sí mismo, se articulan de un modo positivo en el que convergen otros términos como los principios de equidad, democracia, justicia social, derechos humanos, por mencionar algunos y cuyo reenvío entre sí lo configuran como un discurso con un cuerpo sólido, coherente y abarcativo. De este modo, vemos cómo la diversidad pasa a ser un significante vacío, que captura una pluralidad de discursos en cuya significación base sostienen un proyecto político unificado y productivo con un modelo neoliberal. Por significante vacío me refiero a lo propuesto por Laclau (1996), aquel término o configuración discursiva que al ejercer su acción de significante que se vacía de todo vínculo con significantes particulares, "asume el papel de representar el puro ser del sistema $-\mathrm{O}$, más bien el sistema como ser puro y simple- que tal significación es posible" (p. 75). Es decir, el significante vacío actúa como tal en tanto abandone su carácter de diferencial para así asumir una función de representatividad y consolidarse (precariamente) como una relación hegemónica.

Estas relaciones de equivalencia entre diversidad/inclusión/NEE traen consigo una posición de naturalización ante el tránsito que liga las $N E E$ a la inclusión y a la diversidad. En un esfuerzo por diferenciar el concepto de inclusión de aquellas premisas ligadas a lenguajes de normalidad, algunos autores/autoras abordan el paso de integración a inclusión, donde se alude a un cambio conceptual-temporal, en el que la inclusión es situada bajo una mirada de mayor expansión y complejidad del sujeto y su ambiente. No obstante, esta distinción se disemina cuando se vincula con NEE, concepto alineado con lo normativo (Blanco, 2008; Tenorio, 2005) y más aún en el caso de Chile, donde las políticas han optado por restringir el término a discapacidad o al diagnóstico como un modo de abordar La diversidad y resolver las NEE.

Tal como nos señalan Popkewitz y Lindblad (2000), estos estudios se ubicarían en la problemática de equidad, pues su preocupación se centraría en constatar aquello que falta para la inclusión, por lo que desde esta perspectiva resulta innecesario e improductivo cuestionar los discursos oficiales que promueven la inclusión como un propósito a ser cumplido. Así, estos estudios se proponen dar cuenta de deficiencias y generar propuestas que avancen hacia tales metas, como el caso de la recurrente demanda por mejorar la formación inicial (Díaz, 2011; Díaz \& Druker, 2007; Fernández, 2001; Geeregat et al., 2012; Ibáñez, 2010; Julio, 2009; Sánchez et al. 2008; Schmelkes, 2005; Tenorio, 2011), sin dar atención necesariamente a las condiciones de producción de las diferencias educativas. 
Asumir que un cuerpo docente tecnificado, con un saber especializado, con metodologías específicas, abordará de un modo mejor los asuntos relacionados con diferencia, diversidad e inclusión, evidencia una mirada positivista a la que subyace una comprensión del sujeto de carácter fija y natural, de un sujeto/objeto que requiere ciertos modos de tratamiento para ser mejor educado. Esto evidencia una mirada reduccionista ante una realidad compleja en la que se opta por considerar aquello que es más factible manejar, como la tecnificación docente e introducción del saber especializado a través de los profesionales especialistas. Por su parte, Infante (2010) nos llaman a cuestionar los supuestos ontológicos y epistemológicos que sustentan la noción de sujeto, de lo educativo y del rol del profesor, con el propósito de complejizar las temáticas vinculadas a diferencias presentes en el ámbito educativo.

En este sentido, la articulación entre ciertos lenguajes dominantes con sus respectivas corrientes de conocimiento (inclusión, NEE y diversidad) y el desarrollo de políticas, puede ser comprendida como parte de la consolidación del proyecto neoliberal que predomina en Chile. Matus e Infante (2011) analizan tales vínculos y concluyen que mientras las nociones de diversidad estén estrechamente vinculadas a modelos biomédicos asociados al déficit, los usos de diversidad estarán restringidos a la discapacidad y por tanto persistirá una jerarquización estable entre los discursos de normalidad y diferencia. Dichos discursos esencializadores, al ser inscritos en las políticas educativas y en el currículum, definen el modo en el que han de relacionarse y ser configurados las/los sujetos, condiciones que a Matus (2005) la orientan a proponer una imposibilidad para el triunfo de la diversidad.

A modo de ejemplo, las políticas de interculturalidad, son consideradas asimilacionistas, exclusionarias e instrumentalizadoras de ciertos grupos de identidad, en especial de los pueblos originarios (Fernández, 2001; Magendzo, 2000; Williamson, 2005; Zurob, 2009). Con el propósito de democratizar el currículum y hacerlo más participativo, algunos/ algunas proponentes, recurren a los discursos de diversidad, sin cuestionar las premisas que sustentan el currículum actual, cuyos principios se centran en la autonomía, eficacia y éxito académico. En cambio otros/otras reconocen la existencia de tales paradojas y consideran el currículum como un valioso dispositivo de control (Geeregat et al., 2012; Magendzo, 2000; Matus, 2005; Matus \& Infante, 2011; Schmelkes, 2005; Williamson, 2005) у cuya invisibilización de ciertos grupos o valores ligados a etnia, constatan una primacía cultural dominante que privilegia el discurso blanco y masculino (Del Río \& Balladares, 2010).

Asimismo, resulta interesante analizar los usos otorgados a los discursos de diferencia cuando se vinculan con temáticas de sexualidad y género. Por una parte, sin ser una sorpresa, se confirma el escaso desarrollo en investigación que existe en torno a estos asuntos, en contraposición al amplio cuerpo de literatura vinculado a inclusión y diversidad. Por otra parte, respecto del objeto de estudio, en su mayoría los estudios abordan la relación existente entre las diferencias de género y el desempeño/resultados/habilidades académicas y en algunos casos agregan el factor de nivel socio económico (Del Río \& Balladares, 2010; Del Río \& Strasser, 2013; Flores, 2005; Flores, 2007; Madero, 2011), donde si bien las/los autores cuestionan los estereotipos existentes en la escuela y la desigualdad de oportunidades que esto genera, no se cuestionan mayormente los discursos esencialistas y naturalizados que circulan en el ámbito escolar y que al ser asumidos como premisas preestablecidas, más bien tienden a reproducir tales discursos, aun cuando sus resultados aporten en deslegitimar aquellos discursos heteronormativos establecidos desde un lugar de lo incuestionable. 
En un plano similar, estudios relevan la importancia de las prácticas y creencias docentes para entender las desigualdades al interior de la escuela. De este modo, señalan cómo la escuela establece diferencias de género principalmente a través del rol que juegan las/los docentes, quienes reproducen discursos y prácticas sexistas y estereotipadas, lo que se evidencia en los modos de relacionarse y en sus expectativas diferenciadas para las/los estudiantes, que en su generalidad privilegian a los hombres y perjudican a las mujeres (Flores, 2005; Flores, 2007).

En una perspectiva distinta, otras autoras analizan los discursos de diferencia como una producción administrada por diferentes dispositivos, en un caso a través de las políticas públicas (Infante et al., 2011), y en el otro a través de los manuales de enseñanza (Hurtado, 2012). Ambos enmarcan dicha producción en un cuadro político-económico más amplio que posibilita y justifica cierta forma de entender y nombrar a las/los sujetos.

\subsection{PERSPECTIVAS METODOLÓGICAS EN USO: EL DOMINIO CARTESIANO Y LA MASCULINIZA- CIÓN DEL CONOCIMIENTO}

En este apartado me interesa revisar las perspectivas y metodologías de investigación que circulan en la literatura académica, pues asumo la relación imbricada entre perspectivas epistemológicas, selección metodológica y la producción de conocimiento resultante.

Si bien la caracterización nos brinda un panorama actualizado y amplio, resulta más interesante referirnos a dos elementos: la neutralidad aparente de las investigaciones y la relación entre investigador/investigadora e investigada/investigado. Gran parte de las investigaciones asumen un conocimiento neutral, es decir, donde la rigurosidad del método guía las decisiones y permite obtener ciertos conocimientos válidos y, en algunos casos, incluso generales o replicables. Este modo neutral de aproximarse al conocimiento es una de las advertencias que nos propone Bordo (1986), en cuyo planteamiento problematiza las implicancias de la masculinización del conocimiento, donde la relevancia de la legitimidad, rigurosidad y conocimiento válido es asumida bajo ciertos supuestos de certeza y de desfragmentación en orden a lograr la captura o mensurabilidad del mundo.

Vinculado a lo anterior, la relación implicada entre quien investiga y a quien/quienes se investiga, la necesidad de delimitar la posición del sujeto y del objeto de investigación, da cuenta de una mirada modernista/racional e instrumental, donde nuevamente los modos masculinos de pensar el mundo se superponen y se adoptan como garante de un conocimiento serio y valioso.

La mayor parte de los artículos revisados denotan el esfuerzo por permanecer ausentes de la enunciación del conocimiento producido, como si el conocimiento estuviese suspendido en una realidad externa al o la investigadora, esperando a ser capturado. Susan Talburt (1999) y Deborah Britzman (1995), en sus trabajos etnográficos, advertían al campo educacional y criticaban a las/los investigadores por el afán de mantener la creencia de una realidad que está fuera del/la investigadora esperando a ser leída y narrada. Desde otra mirada, estas autoras nos proponen poner atención a los procesos históricos y sociales que forman las experiencias y subjetividades, sin ignorar que tales distinciones son realizadas por el/la investigadora con su propia subjetividad e historia. Por tanto, el conocimiento es producido en base a las intersecciones de experiencias y estructuras sociales de las/los sujetos implicados en ese tiempo, localidad y cultura. 


\section{IDEAS DE AMARRE PARCIAL}

En primer lugar, resulta preocupante, pero no sorprendente, que la mayor parte del cuerpo de la literatura analizada enfrenta las temáticas de diversidad, diferencia e inclusión de un modo tradicional, asumiendo como incuestionables los discursos oficiales dominantes en torno a tales temáticas. Bajo este panorama podemos distinguir un marco discursivo en la literatura académica chilena que privilegia los discursos oficiales de organismos internacionales, un discurso aparentemente neutral, que no cuestiona y más bien naturaliza las diferencias y la normalidad en el ámbito educativo.

Tal como vimos anteriormente, los términos que circulan en el campo académico, se superponen en una suerte de articulación condensada y conforman una red imbricada de lenguajes y conceptos, que funcionan como una cadena nodal, cuya dispersión se puede comprender por su condición de una aparente polisemia que da paso a la ambigüedad que algunos/algunas autoras reclaman. Por otra parte, la articulación antagónica entre los lenguajes vinculados a diversidad e inclusión y los discursos de normalidad, lejos de desplazar a este último, lo reafirman y sostienen al poner al centro de la discusión las diferencias y/o la diversidad, cuya construcción se organiza en torno a la normalidad. Dicho de otro modo, la estrecha vinculación entre los términos antes mencionados y su articulación negada con los discursos de normalidad, configura una red de significados que en su apariencia se comportan como discursos contrarios, no obstante en su juego entretejido constituyen un poder de positividad del sistema que lo configura.

Lo preocupante de tal distinción en el cuerpo de conocimiento revisado es el escaso cuestionamiento respecto de las condiciones de posibilidad y ocurrencia que poseen los discursos en torno a normalidad, puesto que al ser reproducidos dichos discursos oficiales, se instalan como condición natural o inevitable de determinadas culturas o ciertas identidades.

Tal ambigüedad del lenguaje, sumada al dominio cartesiano-masculinizado de la producción de conocimiento, nos conduce a pensar que los discursos que circulan en el contexto educativo reafirman y sostienen un sistema educativo vinculado al proyecto neoliberal, pues la circulación y reproducción de discursos oficiales de organismos internacionales como UNESCO, Banco Mundial, OCDE, por mencionar algunos, trae consigo una serie de principios íntimamente vinculados al desarrollo y progreso de los países (Ahmed, 2007; Baez, 2004; Graham \& Slee, 2007), por lo que hablar de diversidad, inclusión y diferencia desde una posición "neutral", moral, mas no política y centrada en las/los sujetos/objetos "diferentes", converge con los principios neoliberales de aceptación, tolerancia, democracia e igualdad de derechos.

Duggan (2004) y Ahmed (2007), desde distintos centros de interés (sexualidad y género), destacan la articulación entre los discursos de diversidad y neoliberalismo, donde la frontera de inclusión se desplaza bajo intereses económicos, corporativos y morales, pero sin una mirada redistributiva, hacia aquellos sujetos que pueden ser más tolerables que otros (gays, lesbianas, discapacitados, por sobre mapuches, transexuales, entre otros). La escasa presencia de las relaciones de poder en juego, de los intereses y usos políticos otorgados a los asuntos de diversidad, diferencia e inclusión, son algunos de los principales entretejidos discursivos que perpetúan el status quo de los grupos marginados, excluidos o subyugados, en su condición de ser 'otro' 'diferente' en espera a ser incluido por sujetos 'normales-privilegiados'. 
Sobre la base de los trabajos analizados, sostengo que existe un análisis incipiente e insuficiente de la racionalidad de los mismos y un cuestionar reflexivo a la actual manera de comprender ciertos procesos educativos. En este sentido, relevo la necesidad de incorporar otros modos de pensar la investigación educativa, cuestionar la normatividad aun presente en los contextos educativos, los discursos que circulan y aseguran el funcionamiento de tales modos de clasificación. En síntesis, mientras se evite problematizar la normalidad y se persista en neutralizar y naturalizar las diferencias, las/los individuos hasta ahora subalternizados, continuarán sujetos al espacio concedido por la normalidad.

\section{REFERENCIAS BIBIOGRÁFICAS}

Ahmed, S. (2007). The language of diversity. Ethnic and Racial Studies, 30(2), 235-256.

Ainscow, M., \& Sandill, A. (2010). Developing inclusive education systems: The role of organisational cultures and leadership. International Journal of Inclusive Education, 14 (4), 401-416.

Baez, B. (2000). Diversity and its contradictions. Academe-Bulletin of the Aaup, 86(5), 43-47.

Baez, B. (2003). Outsiders within? Academe-Bulletin of the Aaup, 89(4), 41-45.

Baez, B. (2004). The study of diversity: The "knowledge of difference" and the limits of Science. Journal of Higher Education, 75(3), 285-306.

Becerra, S. (2012). Valores de equidad y aceptación en la convivencia de escuelas en contexto indígena: La situación del prejuicio étnico docente hacia los estudiantes mapuche en Chile. Revista de educación, 347, 163-181.

Blanco, R. (2008). Construyendo las bases de la inclusión y la calidad de la educación en la primera infancia. Revista De Educación, 347, 33-54.

Bordo, S. (1986). The Cartesian masculinization of thought. Signs, 11(3), 439-456.

Britzman, D. (1995). The question of belief: writing poststructural ethnography. International Journal of Qualitative Studies in Education, 8(3), 229-238.

Bryan, A. (2010). Corporate multiculturalism, diversity management, and positive interculturalism in Irish schools and society. Irish Educational Studies, 29(3), 253-269.

Buenfil, RN. (1994). Cardenismo: Argumentación y antagonismo en educación. México: Departamento de Investigaciones Educativas Centro de Investigación y de Estudios Avanzados del I.P.N. Consejo Nacional de Ciencia y Tecnología.

Caijao, F. (2005). Educación, escuela y diversidad. Pensamiento Educativo, 37, 27-37.

Davis, L. (1995). Enforcing Normalcy: Disability, Deafness and the Body. London: Verso.

Del Río, F., \& Balladares, J. (2010). Género y Nivel Socioeconómico de los Niños: Expectativas del Docente en Formación. Psykhe, 19(2), 81-90.

Del Río, F., \& Strasser, K. (2013). Preschool children's beliefs about gender differences in academic skills. Sex roles, 68, 231-238.

Deleuze, G. (2006). Diferencia y repetición. Buenos Aires: Amorrortu.

Díaz, N. (2011). Escuela inclusiva: construcción democrática de sociedad en Chile. Revista Iberoamericana de Educación, 55, 2-8.

Díaz, T., \& Druker, S. (2007). La democratización del espacio escolar: Una construcción en y para la diversidad. Estudios pedagógicos, 33, 63-77.

Díaz, T., Figueroa, A., \& Tenorio, S. (2007). Educación de calidad para atender a las necesidades educativas especiales. Una mirada desde la formación inicial docente. REICE: Revista Electrónica Iberoamericana sobre Calidad, Eficacia y Cambio en Educación, 5(5), 109-114.

Duggan, L. (2004). The Twilight of Equality: Neoliberalism, Cultural Politics, and the Attack on Democracy. Boston: Beacon Press.

Duschatzky, S. (1996). De la diversidad en la escuela a la escuela de la diversidad. Propuesta 
Educativa, 7(15), 45-49.

Dussel, I. (2004). Inclusión y exclusión en la escuela moderna argentina: una perspectiva postestructuralista. Cuadernos de Pesquisa, 34(122), 305-335.

Elizalde, A. (2005). Educación, diversidad y cambios de valores. Pensamiento Educativo, 37, 64-83.

Fairclough, N. (2000). Language and neo-liberalism. Discourse \& Society, 11(2), 147-148.

Fairclough, N. (2013). Critical discourse analysis and critical policy studies. Critical Policy Studies, 7(2), 177-197.

Fernández, E. (2001). Un diseño curricular alternativo para las comunidades Aymaras del norte de Chile. Pensamiento Educativo, 37, 277-295.

Flores, R. (2005). Violencia de género en la escuela: sus efectos en la identidad, en la autoestima y en el proyecto de vida. Revista Iberoamericana de Educación, (38), 67-86.

Flores, R. (2007). Representaciones de género de profesores y profesoras de matemática, su incidencia en los resultados académicos de alumnos y alumnas. Revista Iberoamericana de Educación, 43, 103-118.

Foucault, M. (2011). La arqueología del saber. México: Siglo XXI.

Fundación Chile (2013). Análisis de la implementación de los programas de integración escolar en establecimientos que han incorporado a estudiantes con necesidades educativas especiales transitorias. Santiago: MINEDUC.

Graham, L., \& Slee, R. (2007). An illusory interiority: interrogating the discourses/s of inclusion. Educational Philosophy and Theory, 40 (2), 277-293.

Geeregat, O., Vásquez, O., \& Fierro, J. (2012). Procesos de formación inicial docente en contextos multiculturales: inclusión y exclusión. Estudios pedagógicos, 38, 345-351.

Grosz, E. (1995). Space, Time and Perversion: Essays on the Politics of Bodies. London: Routledge. Cátedra.

Haraway, D. (1995). Ciencia, cyborgs y mujeres: la reinvención de la naturaleza. Madrid:

Hurtado, E. (2012). Intelectuales tradicionales, educación de las mujeres y maternidad republicana en los albores del siglo XIX en Chile. Acta literaria, 44, 121-134.

Ibáñez, N. (2010). El contexto interaccional y la diversidad en la escuela. Estudios pedagógicos, $36,275-286$.

Infante, M. (2010). Desafíos a la formación docente: inclusión educativa. Estudios pedagógicos, 36, 287-297.

Infante, M., \& Matus, C. (2009). Policies and practices of diversity: reimagining possibilities for new discourses. Disability \& Society, 24(4), 437-445.

Infante, M., Matus, C., \& Vizcarra, R. (2011). Razonando sobre la idea de diferencia en las políticas educativas chilenas. Universum, 26(2), 143-163.

Julio, C. (2009). Educative diversity in pupil of the system of social protection Shared in common Chile of the commune of Valparaiso: results of an exploratory study. Estudios pedagógicos, $35,93-115$.

Kamp, A., \& Mansouri, F. (2010). Constructing inclusive education in a neo-liberal context: promoting inclusion of Arab-Australian students in an Australian context. British Educational Research Journal, 36(5), 733-744.

Kumashiro, K. (1999). Supplementing normalcy and otherness: Qeer Asian American men reflect on stereotypes identity and oppresion. International Journal of Quealitative Studies in Education, 12 (5), 491-508.

Laclau, E. (1996). Emancipación y diferencia. Madrid: Ariel.

López, V., Julio, C., Morales, M., Rojas, C., \& Pérez, M. (2014). Barreras culturales para la Inclusión: políticas y prácticas de Integración en Chile. Revista de educación, 336, 256-281.

Madero, I. (2011). Inclusión y exclusión de género y clase al interior de la escuela chilena en 4 
comunas del sur de Chile. Estudios pedagógicos, 37 (2), 135-145.

Magendzo, A. (2000). La diversidad y la no discriminación: Un desafío para una educación moderna. Pensamiento Educativo, 26, 173-200.

Magendzo, A. (2005). Alteridad y diversidad: componentes para la educación social. Pensamiento Educativo, 37, 106-116.

Matus, C. (2005). ¿Existe alguna posibilidad que triunfe la diversidad? Pensamiento Educativo, 37, 16-26.

Matus, C., \& Infante, M. (2011). Undoing diversity: knowledge and neoliberal discourses in colleges of education. Discourse: Studies in the Cultural Politics of Education, 32(3), 293-307.

Palaiologou, N., \& Faas, D. (2012). How 'intercultural' is education in Greece? Insights from policymakers and educators. Compare: a Journal of Comparative and International Education, 42(4), 563-584.

Popkewitz, T., \& Lindblad, S. (2000). Educational Governance and Social Inclusion and Exclusion: Some conceptual difficulties and problematics in policy and research. Discourse: Studies in the Cultural Politics of Education, 21(1), 5-44.

Prieto, M. (2001). Educación para la democracia en la escuela. Revista Iberoamericana de Educación, 1-11. Recuperado desde: http://www.rieoei.org/deloslectores/497Prieto.pdf

Rizvi, F., \& Lingard, B. (2013). Globalizing education policy. London: Routledge.

Sánchez, A., Díaz, C., Sanhueza, S., \& Friz, M. (2008). Percepciones y actitudes de los estudiantes de pedagogía hacia la inclusión educativa. Estudios pedagógicos, 34, 169-178.

Schmelkes, S. (2005). Educar en y para diversidad. Pensamiento Educativo, 37, 38-51.

Scott, J. (1992). "Experiencia". En J. Butler, \& J. Scott (Eds.), Feminists Theorize the Political, New York: Routledge.

Seidman, S. (2004). Contested knowledge: Social Theory Today. USA: Blackwell Publishing.

Slee, R. (2008). Rethinking inclusive education. Journal of Intellectual Disability Research, 52, 680-680

Talburt, S. (1999). Open secrets and problems of queer ethnography: Readings from a religious studies classroom. International Journal of Qualitative Studies in Education, 12, 525-539.

Tenorio, S. (2005). La integración escolar en Chile: Perspectiva de los docentes sobre su implementación. Revista Iberoamericana de Investigación Sobre Cambio y Eficacia Escolar, 3, 823831.

Tenorio, S. (2011), Formación inicial docente y necesidades educativas especiales. Estudios pedagógicos, 37, 249-265.

Williamson, G. (2005). Territorios de aprendizaje multiculturales: Transitando a una nueva praxis pedagógica. Pensamiento Educativo, 37, 163-181.

Zurob, C. (2009). "Hare Rapa Nui”: The Rapa Nui boarding School in Viña del Mar, Chile. Education, Emigration and Cultural Diversity. AIBR-Revista de Antropología Iberoamericana, 4, 84-112. 